\title{
Force/Torque Data Modeling for Contact Position Estimation in Peg-in- Hole Assembling Application
}

\author{
Mustafa Waad Abdullah ${ }^{1,2, a}$, Hubert Roth ${ }^{1}$, Michael Weyrich ${ }^{3}$, Jürgen Wahrburg ${ }^{1}$, and Padipat Pluemworasawat ${ }^{1}$ \\ ${ }^{1}$ Institute of Automatic Control Engineering, University of Siegen, Hölderlinstr. 3, Siegen, Germany \\ ${ }^{2}$ Center for Sensor Systems, University of Siegen, Paul-Bonatz Str. 9-11, Siegen, Germany \\ ${ }^{3}$ Institute of Automation and Software Engineering, University of Stuttgart, Pfaffenwaldring 47 Stuttgart, Germany
}

\begin{abstract}
This paper presents an approach for estimating contact positions of peg-in-hole assembling application using data models created from Force/Torque sensor inputs. The assembling task is carried by an industrial robot where the $\mathrm{F} / \mathrm{T}$ sensor is mounted between the robot and the gripper to measure six values of forces and torques during the contact between the peg and the hole. The search approach to estimate the current position of the contact point is implemented based on human like intuitive behavior by maneuvering the peg on the hole's edge. Different models were created and evaluated using experiments results acquired from pervious assembling attempts. Assembling steps, data analysis and models results are presented in this research.
\end{abstract}

\section{Introduction}

Contact force based guiding approaches have attracted the researchers' interest again in the last decay due to the improvement in sensor systems and industrial robots capabilities to carry difficult tasks. This is also reflecting the increased usage of industrial robots in the industry and especially in the automotive sector that share $39 \%$ of total supply according to IFR [1]. However, industrial robots are not being widely enough used in many sectors and one of reasons that can be found more in small and medium enterprises is due to the variety of the given tasks and the environments that requires flexible handling and adaption from the operator [2]. Therefore, fully automated processes could not be achieved unless the industrial robots can meet these dynamic requirements and provide the same flexibility as the human operator does [3]. In addition to that, the industrial robots should be intelligent in sense that they can deal with dynamic environments where offline programming approaches are not suitable. Thus, the solution for this is by integrating external sensors to give the ability to the robots to react to unforeseen events or to interact with human operators and this solution is an ongoing research field.

\section{Problem Description \& State-of-art}

One of the well known challenging application in industry is peg-in-hole assembling. This kind of application, where the tolerance between the peg and hole can be in the range of $0.1 \mathrm{~mm}$, requires delicate environment setup, high movement accuracy, and most importunately is that the parts are in the exact positions where the robot are expecting to find. However, such conditions are hard to maintain, and usually interval calibration for both the work area and the robot are conducted. Because of that, these kinds of applications nowadays are mainly carried by human operators who can quickly recognize and adapt to the changes in parts location or environment.

In order to increase the efficiency of the manufacturing lines, fully automated processes that that reduce processes time and cost are required. Therefore, the research on automated assembling approach is still ongoing with the focus on using industrial robots with additional sensors to replace the human operators. This research uses Force/Torque sensor with industrial robot to carry the automated assembling process. The usage of contact forces for assembling application started more than twenty years ago. First attempts tried to analysis the static contact forces as shown in Fig. 1 (a) in order to find the orientation of the peg to the hole [4]. Some researchers proposed a force/torque map, shown in Fig. 1 (b), which presents the forces and torques on all possible contact positions to find the displacement between peg and hole centers $[5,6]$.

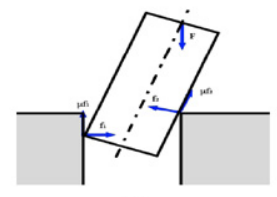

(a)

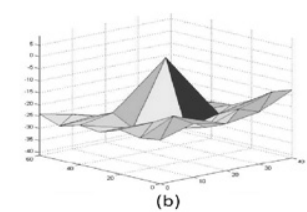

(b)
Figure 1. F/T based Peg-in-Hole Assembling: (a) Static contact force analysis, (b) F/T Map approach.

\footnotetext{
${ }^{\text {a }}$ Corresponding author: abdullah@zess.uni-siegen.de
} 
Since sensor data are very noisy due to the carried weight and the dynamic behavior of the assembling task, a contact state recognition approaches were introduced. This state recognition can be obtained from geometric models as presented in [7]. For the uncertainties problem in the sensor data and also in the position of the parts is addressed in [8] to identify the contact formation in assembling. More recent researches used hidden Markov, Kalman filters with neural network, and maximization based Gaussian to model the assembling task [9, 10, 11]. Tele-operating was proposed [12] to execute the assembling of peg-in-hole. Despite all these efforts, the industry is still lacking for a simple, easy to implement solution for this assembling application. Therefore, this research present a different approach based on human operator handling technique to carry the assembling task as will be described in the next sections.

\section{Position Estimation Approach}

For human operator assembling two parts based on pegin-hole scenario is a simple task. Beside the vision system to align the peg to the hole, the human operator tends to rotate the peg as it touch the surface of the hole edge in order to insert the peg in the direction of the hole center as can be seen below in Fig. 2 .

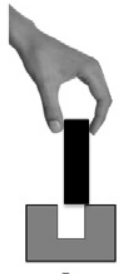

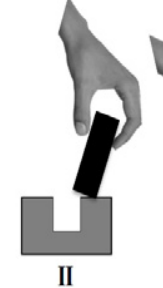

II

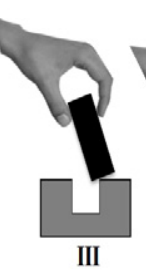

III

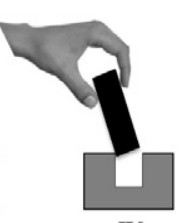

IV

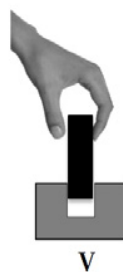

V
Figure 2. Human Operator Approach Steps

In this research, estimating contact position based on the human operator maneuvering as shown in step two and three in the previous figure will be simulated by the industrial robot. Based on force/torque sensor data a model will predict the current contact position between the peg and the hole in order to complete the assembling task.

The proposed approach for maneuvering the peg when it contacts the edge of the hole is shown in the Fig. 3 . First the peg will contact the hole edge, to reach the edge either a predefined position is given to the robot or by using an vision sensor. As soon as a contact is sensed with $\mathrm{F} / \mathrm{T}$ sensor, the robot will rotate on $\mathrm{x}$-axis toward the center of the hole and the values of the $\mathrm{F} / \mathrm{T}$ sensor will be stored in an array. Then the robot will rotate back to the origin orientation, and perform another rotation on $\mathrm{y}$-axis where again the $\mathrm{F} / \mathrm{T}$ sensor values will be stored in the same row of the array.

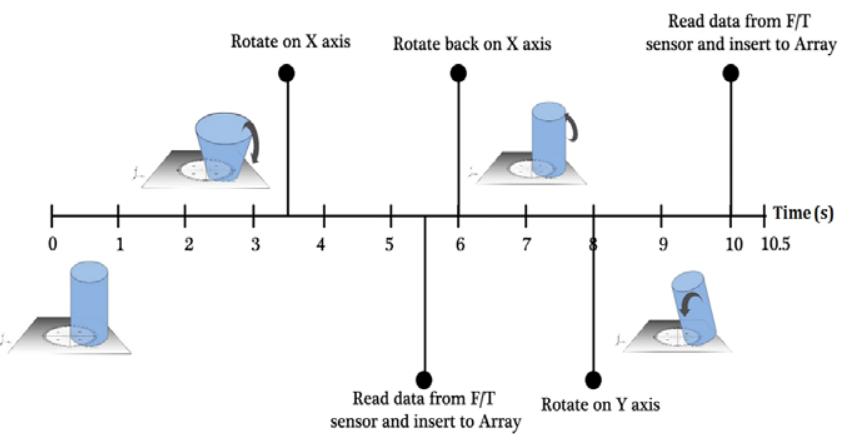

Figure 3. Timeline for Maneuvering on One Contact Point.

The model to predict the position is based on the data collected from the intuitive maneuvering as can be seen in Fig. 4. The F/T data set were collected on each predefined contact position firstly. Then with these collected sets of data, different models are built and tested to compare the prediction position with the actual position from the test data.

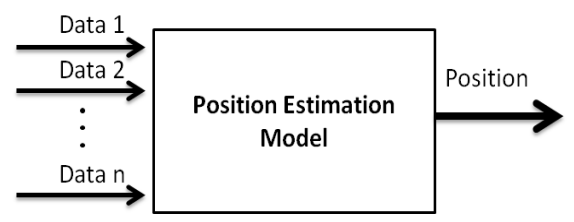

Figure 4. Estimation Model Inputs (Force \& Torques) and Output (Position)

With the best model parameters, the position during the actual assembling can be predicated as soon as live data is collected and treated, and thus, the robot can continue the assembling steps similar to human operator approach explained in Fig. 2.

\section{Experimental Setup}

The industrial robot used in this research is UR5 Robot manufactured by the Danish company Universal Robot and has six degree-of-freedom with $5 \mathrm{~kg}$ payload. The F/T sensor, type ATI Gamma F/T Transducer, provides force measurements of $\pm 32 \mathrm{~N}$ on $\mathrm{x}$ and $\mathrm{y}$ axes, and $\pm 100 \mathrm{~N}$ on $\mathrm{z}$-axis with a resolution up to $1 / 160 \mathrm{~N}$. It also provide torques measurements of $\pm 2.5 \mathrm{Nm}$ on $\mathrm{x}, \mathrm{y}$, and z-axes with resolution up to $1 / 2000 \mathrm{Nm}$. The sensor in mounted between the gripper and the robot to measure the forces and torque applied on the gripper when it touches the objects. As seen in Fig. 5, the hole with 10.1 $\mathrm{mm}$ diameter, in which the robot should make the maneuvering on with a peg of $10 \mathrm{~mm}$ diameter, is divided into four quarters and in each quarter there are nine contact points with 10 degree angle between each other. Thus, a total of 36 contact points are defined to collect the $\mathrm{F} / \mathrm{T}$ data on. 


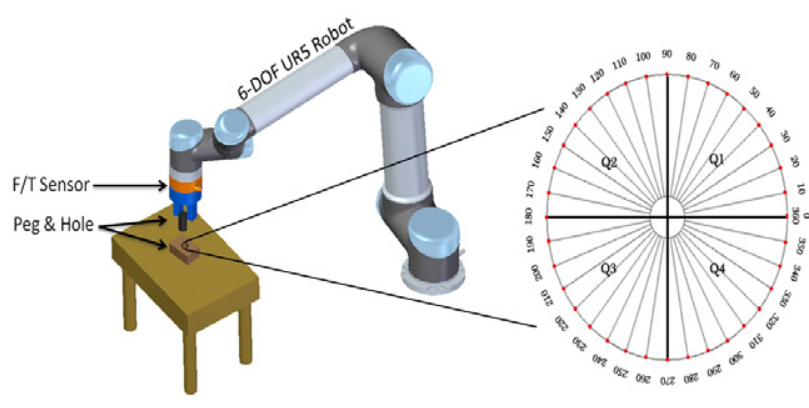

Figure 5. Hardware Setup and Contact Points on Hole's Edge.

For data collection, these predefined contact positions are taught to robot to move vertically to them from a defined home position. 35 sets of data were collected. This means, on each contact point there are 35 different readings each contains 12 values collected from $\mathrm{F} / \mathrm{T}$ sensor, i.e. sensors' six values are stored in each maneuvering as previously explained. An example of the collected data is shown below in Table 1 .
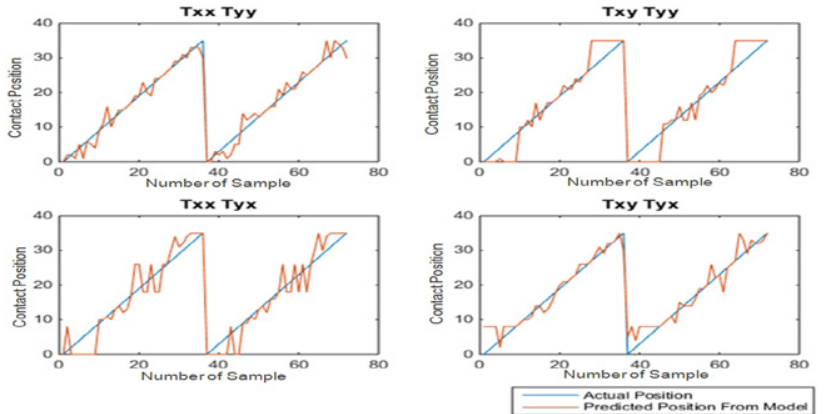

Figure 7. Four Models Positions Estimation Results

From these different models results, it can be seen that based on this intuitive approach, the industrial robot that carries the peg can successfully estimate its current position in regard to the hole. This approach can solve the problem of position uncertainty in object location or repeatability error in industrial robot. Thus, the robot is not required to know the exact location of the hole, and only roughly estimated location that can be sent from a vision sensor, or predefined is sufficient information to

\begin{tabular}{|c|c|c|c|c|c|c|c|c|c|c|c|c|c|}
\hline \multicolumn{1}{|c|}{ TABLE 1 } & \multicolumn{9}{|c|}{ Rotation on X-axis } & \multicolumn{5}{c|}{ Rotation on Y-axis } \\
\hline Pos. & Q. & Fx-x & Fy-x & Fy-x & Tx-x & Ty-x & Tz-x & Fx-y & Fy-y & Fz-y & Tx-y & Ty-y & Tz-y \\
\hline 0 & 1 & 0.7965 & -0.291 & -9.427 & -0.040 & -0.128 & -0.002 & 1.4576 & 0.0094 & -13.15 & 0.0085 & -0.206 & -0.002 \\
1 & 1 & 0.2329 & -0.367 & -7.836 & -0.047 & -0.072 & 0.0072 & 1.4572 & 0.3028 & -9.229 & 0.0317 & -0.076 & -0.004 \\
\hline 2 & 1 & 0.1913 & -0.077 & -5.674 & -0.019 & -0.058 & 0.0016 & 0.0304 & 0.5590 & -8.296 & 0.0535 & -0.034 & -0.005 \\
\hline 3 & 1 & 0.1206 & 1.3433 & -5.043 & 0.1233 & -0.026 & -0.009 & 0.5936 & -0.082 & -3.571 & -0.002 & -0.038 & -0.002 \\
\hline 4 & 1 & -0.160 & 1.4322 & -5.427 & 0.125 & -0.008 & -0.003 & 0.0292 & 0.0737 & -3.892 & 0.0116 & 0.011 & -0.002 \\
\hline$\ldots$ & $\ldots$ & $\ldots$ & $\ldots$ & $\ldots$ & $\ldots$ & $\ldots$ & $\ldots$ & $\ldots$ & $\ldots$ & $\ldots$ & $\ldots$ & $\ldots$ & $\ldots$ \\
\hline
\end{tabular}

\section{Data Modeling \& Results}

Different models were created and tasted based on different inputs using MATLAB Fitting Tool. Firstly, models with the average of the collected data set where created. In this approach, the estimation models can have either a single input, ex. torque on $\mathrm{x}$-axis $\left(\mathrm{T}_{\mathrm{x}}\right)$, or multiple inputs such as torque and force $\left(T_{x}\right.$ and $\left.F_{y}\right)$. From the experiment results, and also based on the rotation of peg on $\mathrm{x}$ and $\mathrm{y}$ axes, the torques on these axes as shown in Fig. 6 are the ones that can give more accurate estimation due to this maneuvering. The best model to predict the contact position are based on input torques on $\mathrm{x}$ and $\mathrm{y}$ axes as can be seen in Fig. 7 and with multiple data inputs.
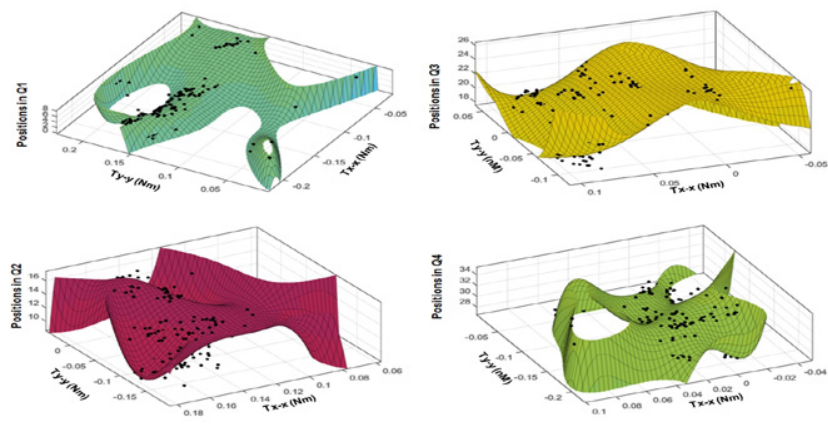

Figure 6. Multiple Data Inputs Based Models successfully carry the assembling task based on estimation the contact position.

\section{References}

1. Executive Summery World Robotics 2013, Industrial Robots, International Federation of Robotics-IFR (2014).

2. A. Blomdell, G. Bolmsjö, T. Brogardh, and others,: Extending an Industrial Robot Controller, Implementation and Applications of a Fast Open Sensor Interface. IEEE Robotics and automation Magazine (September 2005).

3. E. Kus, R. Grüninger and R. Hüppi: Integration of Intelligent Sensors for Sensor guided Motions in Industrial Robot Applications. IEEE International Conference on Automation and Logistics Qingdao, (China 2008).

4. I.-W. Kim, Active Peg-in-hole of Chamferless Parts using Force Moment Sensor, IEEE (1999).

5. W.S. Newman, Y. Zhao, and Y. Pao: Interpretation of Force and Moment Signals for Compliant Peg-inHole Assembly. 2001IEEE International Conference on Robotics \& Automation, (Korea 2001).

6. Dietrich, F., Wobbe, F., and Others: Enhancements of force-torque map based assembly applied to parallel robots. International Conference on Industrial Technology, (Chile 2010).

7. Hirai S., Iwata K.: Recognition of contact state based on geometric model. International conference on robotics and automation (France1992). 
8. Farahat A., Graves B., Trinkle J.: Identifying contact formations in the presence of uncertainty. International conference intelligent robotics systems, Pittsburgh (USA 1995).

9. Lau HY.: A hidden Markov model-based assembly contact recognition system. Mechatronics: the science of intelligent machines, volume (13), page: 1001-1023 (2003).

10. Cortesao, R., Koeppe, R., and Others: Data fusion for robotic assembly tasks based on human skills. IEEE Transactions on Robotics, volume (20), pages: 941-952. (2004).
11. Savarimuthu, T. R., Liljekrans, D., and Ellekilde, L.: Analysis of human peg-in-hole executions in a robotic embodiment using uncertain grasps. International workshop on robot motion and control (Poland 2013).

12. Jasim, I. F., Plapper, W. Contact-state monitoring of force-guided robotic assembly tasks using expectation maximization-based Gaussian mixtures models. The international journal of advanced manufacturing technology, volume 73, pages: 623633. (2014). 